\title{
Marine Ranching
}

$\mathrm{E}$ nvironmental change and human activities have caused habitat degradation and resource decline in China's coastal waters. Chinese scientists have been seeking to contain these damages and finally restore the coastal ecosystem where marine organisms can habit and prosper, expecting rewards such as a good supply of high-quality seafoods to the table.

To fulfill the task, scientists at the Institute of Oceanology. Chinese Academy of Sciences (IOCAS) resorted to the concept of "Narine ranching". Aso known as sea farming. the technology was developed in the 1970s. It is a type of aquaculture involving the cultivation of marine organisms for food and other products in open sea or in an enclosed section of coastal waters.

At the beginning. they experimented such concept in two coastal locations, the Jiaozhou Bay (Shangdong) and the Daya Bay (Guangdong). The experiment initiates strain breeding and the healthy aquaculture of shrimp. sea cucumber. shellfish, algae etc.. and establishes a reproducible and scalable paradigm of marine ranching. providing a solid technical support for increasing fishermen's income, enhancing fishery efficiency, and upgrading the aquaculture industry.

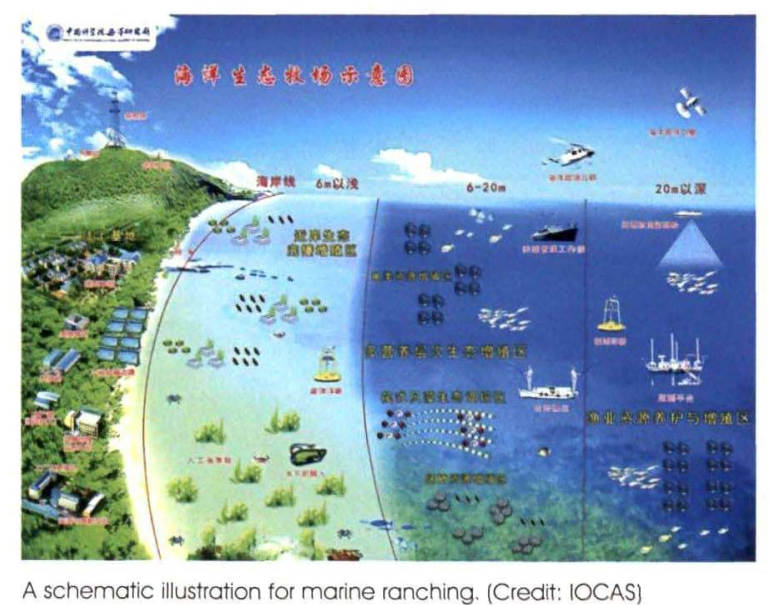

A schematic illustration for marine ranching. (Credit: IOCAS)

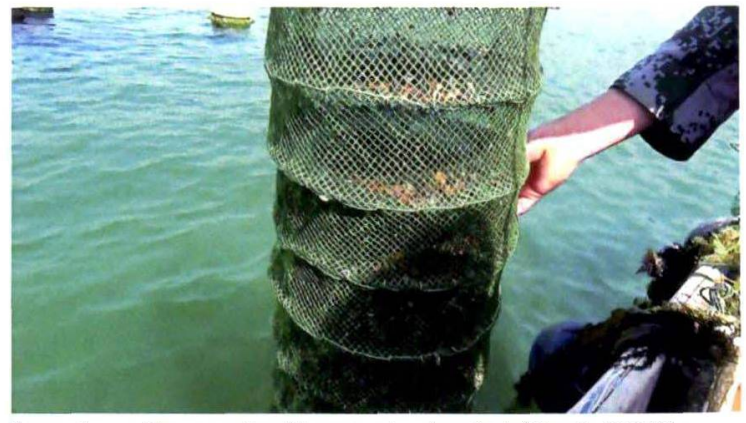

The culture of bay scallop (Argopecten irradias). (Credit: IOCAS)

Among the many achievements, a particular one entitled -Research and Application of Bay Scallop (Argopecten irradias) Introduction. Breeding and Cultivation" stands out. winning the first prize of the National Science and Technology Progress Award in 1990.

Along these explorations. IOCAS scientists developed the key techmologies and facilities for marine ecological environment construction and established the construction standards for marine ranches. They also made strategic shifts at many levels - restoring marine ecosystem from local repairing to systematic rebuilding. developing biological resources from production restoration to ecological restoration. and building new environmental technologies from single monitoring and evaluation to comprehensive early warning and forecast.

From 2015 to 2017. IOCAS scientists constructed five demonstration bases of marine ranching in such locations as Dalian, Tangshan. Yantai and Rizhao. They found that these demonstration bases can greatly improve the ecological environment and stabilized the ecosystem in the close waters. judging by their first-class water quality in the core area and enhanced aquatic biodiversity and abundance. These marine ranches have not only brought about a huge economic bonus for the local fishermen by doubling the average annual income. but also fueled a more sustainable and efficient marine fisheries. 